\title{
Myocardial strain and refractory angina: an intriguing puzzle
}

\author{
Antonio Vitarelli ${ }^{1,2}{ }^{10}$
}

Received: 3 June 2021 / Accepted: 4 June 2021 / Published online: 2 July 2021

(c) The Author(s), under exclusive licence to Springer Nature B.V. 2021

Refractory angina (RA) refers to chronic angina-type chest pain (persistence for more than 3 months) associated with reversible ischaemia in the presence of obstructive coronary heart disease (CAD), which cannot be controlled by pharmacological therapy, bypass grafting or percutaneous coronary interventions, including chronic total occlusion $[1,2]$. Due to an ageing population, and improved survival from CAD, the number of RA "no option" patients is growing. Microvascular dysfunction, alone or in association with epicardial coronary artery disease, is thought to contribute significantly to refractory angina, although most of the techniques used in the treatment of refractory angina have not been specifically tested in patients with microvascular dysfunction. Physical and psychological components of RA may require pharmacological and psychological strategies along with interventional approaches such as external counterpulsation, coronary sinus constriction, and autologous cell therapy.

External counterpulsation (ECP), also known as enhanced external counterpulsation (EECP), has been proposed as a noninvasive procedure that aims to improve cardiac function in patients with refractory angina [3-5]. It uses serial inflation and deflation of three sets of lower-extremity cuffs timed to the patient's electrocardiogram, providing diastolic augmentation of the coronary artery perfusion pressure, systolic unloading, reduced aortic impedance, and increased venous return and cardiac output, thus similar hemodynamic effects to the intraaortic balloon pump. The cyclic inflation/ deflation leads to shear stress on the vascular endothelium as in moderate intensity aerobic exercise, enhancing myocardial supply by opening dormant collaterals, promoting angiogenesis and decreasing ischemia, and reducing myocardial

This comment refers to the article available online at https://doi. org/10.1007/s 10554-021-02289-x

Antonio Vitarelli

vitar@tiscali.it; cardiodiagnostica@gmail.com

1 Department of Medicine and Cardiology, Sapienza

University, Rome, Italy

2 Cardiodiagnostica, Via Lima 35, 00198 Rome, Italy oxygen demand through the increase of plasma nitric oxide and decrease of the level of endothelin with improvement of peripheral endothelial function. This dual mechanism of action, a central mechanism that stimulates angiogenesis and a peripheral mechanism that reduces the stiffness of both elastic and muscular arteries, has been shown to improve the Canadian Cardiovascular Society clinical class of angina and exercise tolerance of patients. This therapy is also currently being studied as a treatment for patients with congestive heart failure. Therefore echocardiographic methods enabling to evaluate left ventricular (LV) function should be clinically useful in this context.

Novel echocardiographic techniques allow to assess myocardial deformation (strain) and quantify its multiplanar and multidirectional components, including longitudinal, radial and circumferential contraction (leading to longitudinal, radial and twisting deformation), instead of providing only a global index of left ventricular function as is the case with the ejection fraction [6]. The complex systolic and diastolic deformation of the left ventricular myocardium results from the contraction and relaxation of various groups of fibres. LV subendocardial and subepicardial fibres are arranged in a longitudinal manner, making a spiral around the ventricular chamber (clockwise oriented subepicardial, and counterclockwise oriented subendocardial), whereas mid myocardial fibres are oriented circumferentially. Inclusion of strain imaging as assessed by speckle-tracking echocardiography, primarily LV global longitudinal strain, is frequently used in clinical practice and has recently been recommended in major guidelines.

Experimental and clinical studies have shown that deformation extent is influenced by loading conditions and heart rate and strain changes are found across a broad spectrum of heart disease such as valvular heart disease, congenital heart disease, ischemic heart diseae, and cardiomyopathies (Fig. 1). Long-lasting volume overload in congenital and/or valvular heart disease causes progressive chamber enlargement and increasing wall stress that can result in contractile dysfunction, decreased longitudinal strain values and, later, reduced ejection fraction. Globally impaired ventricular 
Fig. 1 Factors influencing myocardial strain in different heart diseases

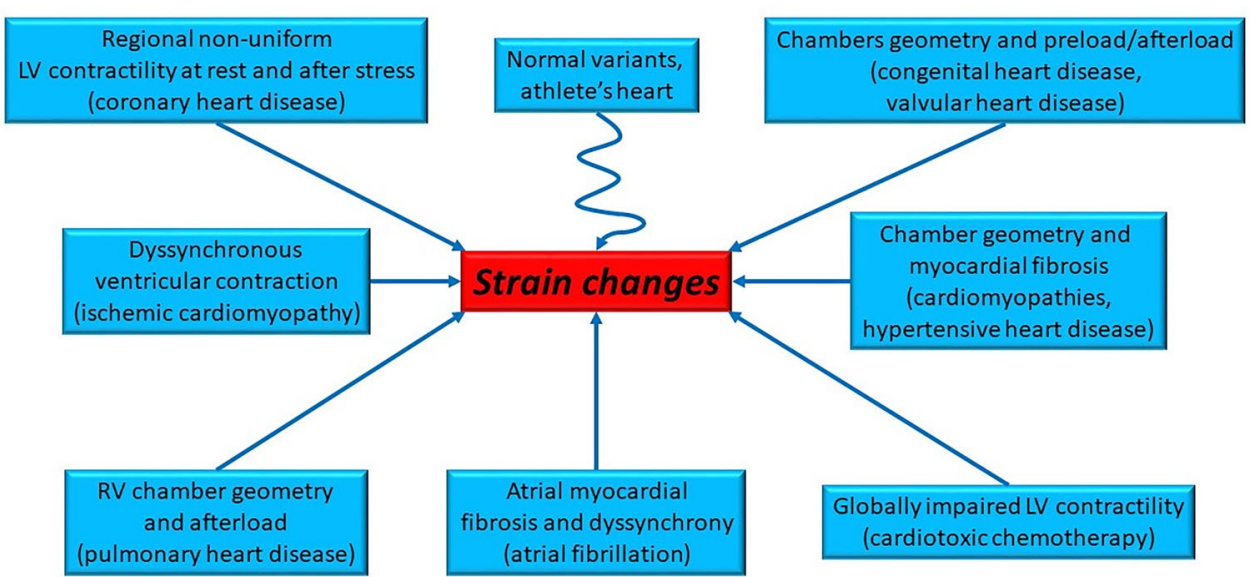

function with reduced strain parameters can occur after chemotherapy or in systemic disease. Non-homogeneous timing of contraction can be observed in ischemic cardiomyopathy before resynchronization therapy.

In coronary artery disease, the onset of acute ischemia leads in seconds to decreased systolic shortening associated with post-systolic shortening (PSS) after aortic valve closure in risk areas [6-12]. Since in the dysfunctional myocardium radial thickening and inward endocardial movement predominate, longitudinal strain regional changes do not automatically correspond to the visual impression of wall motion. Post-systolic shortening (Fig. 2) is observed mainly during isovolumic relaxation and can be considered an active component of ventricular "relaxation" phase so that the ischemic area can shorten during LV pressure drop while the surrounding tissue is relaxing. A segment showing systolic hypokinesia/akinesia and postsystolic shortening is assumed to be actively contracting and therefore potentially viable. If the ischemia lasts longer, the ischemic region undergoes a systolic stretch and the subsequent post-systolic shortening can rather be interpreted as an index of passive recoil due to the interaction between the ischemic and surrounding myocardium [9]. Newly occurring post-systolic shortening during dobutamine stress echocardiography may suggest induced ischemia. Post-systolic contraction occurs during an energetically demanding and vulnerable phase of the cardiac cycle (closely connected with the underlying discrepancy between production and consumption of adenosine triphosphate-ATP-), and its persistence may be due to metabolic stunning (or ischemic memory). However, PSS is a non-specific sign of regional dysfunction [10] and can also be caused by heterogeneity of contraction among myocardial regions as it happens in chronic ischemic scar and in other diseases that may present with regional fibrosis such as hypertrophic or dilated cardiomyopathies and hypertensive heart disease. Minor post-systolic shortening is often found in approximately $30 \%$ of myocardial segments in healthy hearts in the presence of normal systolic function and low strain magnitude. Physiological PSS has a shorter delay in relation to aortic valve closure compared to pathological PSS. The presence of PSS observed in healthy subjects [13] in myocardial segments that have initiated the relaxation process suggested the existence of an additional delayed active contraction after aortic valve closure which occurs later due to its location in the epicardial region. PSS in normal individuals was most frequently observed in the interventricular septum and basal segments, and this was interpreted as the origin of the active contractile movement which leads to LV protodiastolic longitudinal elongation and untwisting provoking diastolic suction.

In the present issue of the International Journal of Cardiovascular Imaging [14], Pravian et al. assessed 43 patients with refractory angina who could not be revascularized conventionally. They were randomized into two groups, the External Counterpulsation (ECP) group and the Sham/control group. ECP therapy was given for thirtyfive sessions. 2DSTE data, including global longitudinal strain (GLS), post-systolic shortening (PSS) and post-systolic index (PSI), were obtained before and after therapy. Thirtyfive ECP therapy sessions did not affect either global or segmental left ventricular mechanical function in patients with refractory angina, but the mechanical function of myocardial segments with PSS improved after ECP therapy. PSS was regarded as present if PSI $\geq 20 \%$. PSS persisted after brief periods of ischemia, reflecting a state of myocardial stunning that can be used to identify ischemic memory and suggesting it may be a marker for functional recovery after ischemic events.

This interesting study is the first to assess left ventricular mechanical function using longitudinal strain and postsystolic shortening in patients who have a full treatment of 35 ECP sessions. These patients had refractory angina which was not ideal for revascularization and low strain values suggesting myocardial dysfunction and poor prognosis. The reason the authors found no difference in GLS between patients 
Fig. 2 Representative longitudinal strain speckle-tracking curves in apical 4-chamber views. A Global longitudinal strain in a healthy subject. B Reduced strain and segmental post-systolic shortening in a patient with coronary artery disease. $A V C$ aortic valve closure, ESL early-systolic lengthening, ESS end-systolic strain, $G S=G L S$ global longitudinal strain (average), IVRT isovolumic relaxation time, $P S S$ post-systolic shortening. PSI (post-systolic index) (calculated segmentally $)=[($ peak global longitudinal strain - peak systolic longitudinal strain)/ (peak global longitudinal strain) $] \times 100 \%$
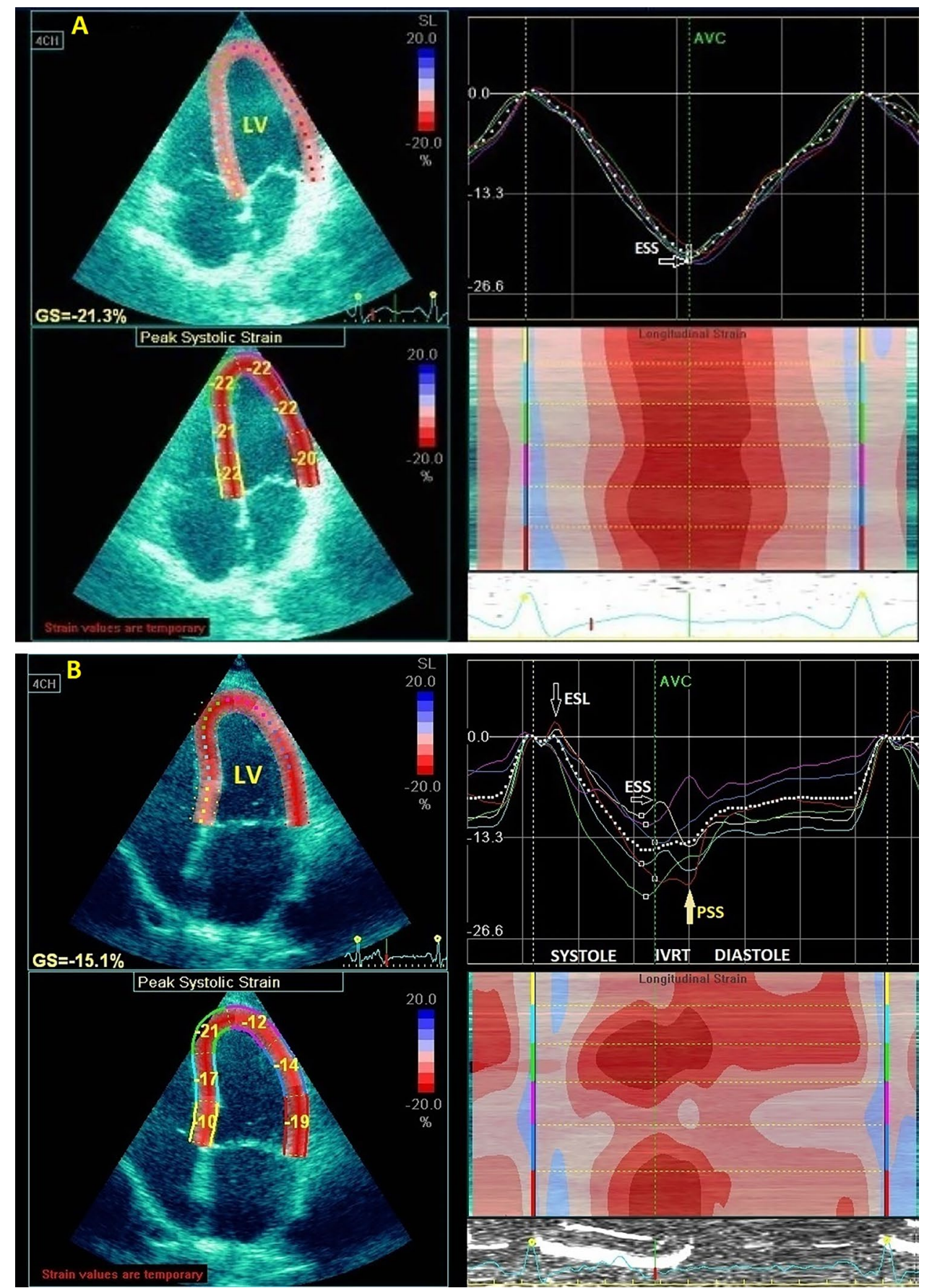

and controls is that adequate revascularization, although a strong stimulating factor in ischemic tissue regeneration, had a limited effect on reduced contractile proteins during myocardial hibernation. Failure to improve this metabolic status may contribute to persistent myocardial dysfunction and delayed recovery of myocardial function.

There are few previous studies dealing with LV function during ECP therapy. Controversial results on left ventricular ejection fraction (LVEF) after ECP sessions were obtained, showing a significant difference or no difference in LVEF between before and after ECP. In some studies ECP treatment did not alter systolic LV function but significantly improved the parameters of ventricular diastolic function, whereas other authors reported significant differences in LV systolic function between pre- and post-ECP therapy measurements [15]. Currently, 2DSTE method is considered superior to Doppler and volumetric methods in evaluating subclinical left ventricular dysfunction. ECP was found to benefit CAD patients by improving global and regional LV systolic and diastolic function according to velocity, strain, 
and strain rate imaging [16]. Changes in mechanical parameters (global longitudinal strain) during a one-hour session of ECP therapy has been reported in patients with refractory angina [17] but persistence of mechanical improvement after completion of 35 ECP therapy sessions was not assessed.

In the study of Pravian et al., the data from sub-analysis showed that segments with pathological PSS patterns tended to have left ventricular mechanical improvement with ECP therapy for 35 sessions. Authors suggest these findings have some clinical implications. Firstly, the baseline magnitude of longitudinal strain before ECP therapy cannot be used as the only parameter to predict improvement of the mechanical function after ECP, albeit better strain values may reflect fewer fibrotic myocardial areas. This may occur because the greatest reduction in longitudinal deformation can be observed not only in the fibrotic myocardium but also in some ongoing ischemic myocardial regions, especially when associated with PSS. Secondly, PSS pattern before ECP therapy may help in selecting appropriate patients who could benefit from ECP. A further trial to determine the minimum number of PSS segments that provide clinical and mechanical benefit from ECP treatment is auspicated.

Still some points deserve a specific comment. This study assessed the outcome just once after completion of ECP therapy, so persistent effect was not known. Persistence of subtle abnormal myocardial deformation such as post-systolic shortening after transient ischemia can help in diagnosing a history of myocardial ischemia (myocardial ischemic memory) but the mechanisms of persistence have not been completely clarified. Three-dimensional (3D) echocardiography allows to analyze wall motion in all LV segments in one image acquisition without being affected by the through-plane motion and assess the spatial extent of wall motion abnormalities more accurately than two-dimensional echocardiography. Recently, in a dog model with 2-min coronary occlusion followed by reperfusion [18], it was found that the combined parameter of early systolic lenghtening and post-systolic shortening detected by 3D speckle-tracking echocardiography (3DSTE) provided better diagnostic accuracy than PSS alone in detecting subtle changes in myocardial deformation caused by systolic stunning (ischemic memory) whereas it could be hard to identify mild changes in peak systolic strain due to its relatively large normal variation. Though these data were acquired from transepicardial echocardiography in open-chest dogs and feasibility in patients could be lower, 3D speckle-tracking echocardiography appeared advantageous for the assessment of spatial extent of ischemic memory because rapid data acquisition after the ischemic episode was useful in detecting ischemic memory and estimating the culprit vessel and risk area. Technical advances in echocardiography will enable imaging at a very high frame rate to provide more insight into cardiac blood flow properties and myocardial contractility, especially during the very short-lived isovolumetric contraction and relaxation periods. A further limitation of the study of Pravian et al. was not using dobutamine stress echocardiography (DSE) to detect segments with stress-induced ischemia. The assessment of 2DSTE systolic strain or postsystolic shortening during early recovery after DSE helps in identifying significant CAD compared with conventional visual analysis after STsegment elevation myocardial infarction [19]. Some studies have shown that 2DSTE has high sensitivity and specificity for early identification of viable myocardium in patients with acute and old myocardial infarction [20]. Diagnostic accuracy of visual and strain analysis was compared, and expertise was shown to affect strain assessment to detect significant coronary artery disease during DSE [21]. The accuracy of speckle tracking echocardiography to assess myocardial deformation in a large range of heart rates and strain magnitudes compared to sonomicrometry was also demonstrated in vitro [22]. However, albeit speckle tracking appears to be a reliable and reproducible technique to analyze myocardial deformation variations during stress echocardiography, further investigation is warranted to assess the impact of angulated and artefactual outof-plane motions and inter-vendor variability. Last, the evaluation of the elastic properties of the aorta during ECP therapy is an interesting issue that focuses on the pathophysiological link between myocardial function and aortic strain. It has been shown that ECP therapy decreases central and peripheral arterial stiffness, which may explain improvements in myocardial oxygen demand in patients with chronic angina pectoris after treatment [4]. These findings are in keeping with other studies showing that afterload-increasing maneuvers may enable the revelation of concealed ischemic memory and mild afterload augmentation allowed the reappearance of PSS that had disappeared after transient ischemia [23].

In summary, in patients with refractory angina who are not ideal for revascularization the intrinsic myocardial function in segments with PSS patterns tends to improve after standard protocol of ECP therapy sessions. Future studies using strain parameters, three-dimensional speckle tracking, high frame rate echocardiography and dobutamine stress imaging will certainly help to work out more detailed criteria for defining viable myocardium.

\section{Declarations}

Conflict of interest The author has no conflict of interests to disclose.

Ethical approval This editorial comment is compliant with ethical standards. 


\section{References}

1. Knuuti J, Wijns W, Saraste A, Capodanno D, Barbato E, FunckBrentano C, Prescott E, Storey RF, Deaton C, Cuisset T, Agewall S, Dickstein K, Edvardsen T, Escaned J, Gersh BJ, Svitil P, Gilard M, Hasdai D, Hatala R, Mahfoud F, Masip J, Muneretto C, Valgimigli M, Achenbach S, Bax JJ, ESC Scientific Document Group (2020) 2019 ESC Guidelines for the diagnosis and management of chronic coronary syndromes. Eur Heart J 41(3):407-477. https:// doi.org/10.1093/eurheartj/ehz425

2. Davies A, Fox K, Galassi AR, Banai S, Ylä-Herttuala S, Lüscher TF (2021) Management of refractory angina: an update. Eur Heart J 42(3):269-283. https://doi.org/10.1093/eurheartj/ehaa820

3. Caceres J, Atal P, Arora R, Yee D (2021) Enhanced external counterpulsation: a unique treatment for the "No-Option" refractory angina patient. J Clin Pharm Ther 46(2):295-303. https://doi.org/ 10.1111/jcpt. 13330

4. Casey DP, Beck DT, Nichols WW, Conti CR, Choi CY, Khuddus MA, Braith RW (2011) Effects of enhanced external counterpulsation on arterial stiffness and myocardial oxygen demand in patients with chronic angina pectoris. Am J Cardiol 107(10):1466-1472. https://doi.org/10.1016/j.amjcard.2011.01.021

5. Wu E, Mårtensson J, Desta L, Broström A (2021) Predictors of treatment benefits after enhanced external counterpulsation in patients with refractory angina pectoris. Clin Cardiol 44(2):160167. https://doi.org/10.1002/clc.23516

6. Voigt JU, Cvijic M (2019) 2- and 3-dimensional myocardial strain in cardiac health and disease. JACC Cardiovasc Imaging 12(9):1849-1863. https://doi.org/10.1016/j.jcmg.2019.01.044

7. Vitarelli A, Montesano T, Gaudio C, Conde Y, Cimino E, Dangeli I, Dorazio S, Stellato S, Battaglia D, Padella V, Caranci F, Ciancamerla M, Di Nicola AD, Ronga G (2006) Strain rate dobutamine echocardiography for prediction of recovery after revascularization in patients with ischemic left ventricular dysfunction. J Card Fail 12(4):268-75. https://doi.org/10.1016/j.cardfail.2006.02.003

8. Nguyen BL, Capotosto L, Persi A, Placanica A, Rafique A, Piccirillo G, Gaudio C, Gang ES, Siegel RJ, Vitarelli A (2015) Global and regional left ventricular strain indices in post-myocardial infarction patients with ventricular arrhythmias and moderately abnormal ejection fraction. Ultrasound Med Biol 41(2):407-417. https://doi.org/10.1016/j.ultrasmedbio.2014.09.025

9. Skulstad H, Edvardsen T, Urheim S, Rabben SI, Stugaard M, Lyseggen E, Ihlen H, Smiseth OA (2002) Postsystolic shortening in ischemic myocardium: active contraction or passive recoil? Circulation 106(6):718-724. https://doi.org/10.1161/01.cir.00000 24102.55150.b6

10. Voigt JU, Lindenmeier G, Exner B, Regenfus M, Werner D, Reulbach U, Nixdorff U, Flachskampf FA, Daniel WG (2003) Incidence and characteristics of segmental postsystolic longitudinal shortening in normal, acutely ischemic, and scarred myocardium. J Am Soc Echocardiogr 16(5):415-423. https://doi.org/10.1016/ s0894-7317(03)00111-1

11. Brainin P, Hoffmann S, Fritz-Hansen T, Olsen FJ, Jensen JS, Biering-Sørensen T (2018) Usefulness of postsystolic shortening to diagnose coronary artery disease and predict future cardiovascular events in stable angina pectoris. J Am Soc Echocardiogr 31(8):870-879.e3. https://doi.org/10.1016/j.echo.2018.05.007

12. Brainin P, Biering-Sørensen SR, Møgelvang R, de Knegt MC, Olsen FJ, Galatius S, Gislason GH, Jensen JS, Biering-Sørensen T (2019) Post-systolic shortening: normal values and association with validated echocardiographic and invasive measures of cardiac function. Int J Cardiovasc Imaging 35(2):327-337. https:// doi.org/10.1007/s10554-018-1474-2

13. Mora V, Roldán I, Romero E, Saurí A, Romero D, Pérez-Gozalbo J, Ugalde N, Bertolín J, Rodriguez-Israel M, Delgado CP,
Lowenstein JA (2018) Myocardial contraction during the diastolic isovolumetric period: analysis of longitudinal strain by means of speckle tracking echocardiography. J Cardiovasc Dev Dis 5(3):41. https://doi.org/10.3390/jcdd5030041

14. Pravian D, Soesanto AM, Ambari AM, Kuncoro BRMAS, Dwiputra B, Muliawan HS, Sukmawan R (2021) The effect of external counterpulsation on intrinsic myocardial function evaluated by speckle tracking echocardiography in refractory angina patients: a randomized controlled trial. Int J Cardiovasc Imaging. https:// doi.org/10.1007/s10554-021-02289-x

15. Arora RR, Lopez S, Saric M (2005) Enhanced external counterpulsation improves systolic function by echocardiography in patients with coronary artery disease. Heart Lung 34(2):122-125. https://doi.org/10.1016/j.hrtlng.2004.06.007

16. Esmaeilzadeh M, Khaledifar A, Maleki M, Sadeghpour A, Samiei N, Moladoust H, Noohi F, Haghighi ZO, Mohebbi A (2009) Evaluation of left ventricular systolic and diastolic regional function after enhanced external counter pulsation therapy using strain rate imaging. Eur J Echocardiogr 10(1):120-126. https://doi.org/10. 1093/ejechocard/jen183

17. Eftekhari A, May O (2012) The immediate hemodynamic effects of enhanced external counterpulsation on the left ventricular function. Scand Cardiovasc J 46(2):81-86. https://doi.org/10.3109/ 14017431.2012 .654814

18. Kozuma A, Asanuma T, Masuda K, Adachi H, Minami S, Nakatani S (2019) Assessment of myocardial ischemic memory using three-dimensional speckle-tracking echocardiography: a novel integrated analysis of early systolic lengthening and postsystolic shortening. J Am Soc Echocardiogr 32(11):1477-1486. https:// doi.org/10.1016/j.echo.2019.06.013

19. Joyce E, Hoogslag GE, Al Amri I, Debonnaire P, Katsanos S, Bax JJ, Delgado V, Marsan NA (2015) Quantitative dobutamine stress echocardiography using speckle-tracking analysis versus conventional visual analysis for detection of significant coronary artery disease after ST-segment elevation myocardial infarction. J Am Soc Echocardiogr 28(12):1379-89.e1. https://doi.org/10. 1016/j.echo.2015.07.023

20. Garcia MJ, Kwong RY, Scherrer-Crosbie M, Taub CC, Blankstein R, Lima J, Bonow RO, Eshtehardi P, Bois JP (2020) American Heart Association Council on Cardiovascular Radiology and Intervention and Council on Clinical Cardiology. State of the Art: Imaging for Myocardial Viability: a Scientific Statement From the American Heart Association. Circ Cardiovasc Imaging 13(7):e000053. https://doi.org/10.1161/HCI.0000000000000053

21. Yang LT, Kado Y, Nagata Y, Otani K, Otsuji Y, Takeuchi M (2017) Strain Imaging with a bull's-eye map for detecting significant coronary stenosis during dobutamine stress echocardiography. J Am Soc Echocardiogr 30(2):159-167.e1. https://doi.org/10. 1016/j.echo.2016.10.011

22. Hodzic A, Chayer B, Wang D, Porée J, Cloutier G, Milliez P, Normand H, Garcia D, Saloux E, Tournoux F (2018) Accuracy of speckle tracking in the context of stress echocardiography in short axis view: an in vitro validation study. PLoS ONE 13(3):e0193805. https://doi.org/10.1371/journal.pone.0193805

23. Masuda K, Asanuma T, Sakurai D, Nakatani S (2018) Afterload augmentation can reveal concealed myocardial ischemic memory. JACC Cardiovasc Imaging 11(11):1727-1729. https://doi.org/10. 1016/j.jcmg.2018.03.022

Publisher's Note Springer Nature remains neutral with regard to jurisdictional claims in published maps and institutional affiliations. 